\title{
El servicio de energía eléctrica, un análisis a nivel nacional y provincial
}

\section{Introducción}

La energía eléctrica es uno de los servicios básicos más usados en el mundo, y al que todos deben tener acceso. A través del tiempo, Ecuador ha mejorado la infraestructura eléctrica tanto en producción como en distribución, para satisfacer la demanda local de electricidad; es así, que se construyen nuevos proyectos hidroeléctricos como: Coca-Codo-Sinclair, Toachi Pilatón, Quijos, entre otros.

En Tungurahua, así como en las provincias de Pastaza, Napo, Morona Santiago y recientemente Bolívar, la institución que se encarga del suministro de energía eléctrica es la Empresa Eléctrica Ambato Regional Centro Norte S.A. - EEASA.

El presente artículo realiza un análisis de la evolución en la producción, demanda, cobertura, pérdida y precios de la energía eléctrica a nivel nacional y en Tungurahua utilizando datos de: EEASA, Agencia de Regulación y Control de Electricidad - ARCONEL, Consejo Nacional de Electricidad - CONELEC y Sistema Nacional de Información - SNI.

\section{Producción de energía eléctrica}

A nivel nacional la producción total de energía eléctrica va en aumento, refleja una tasa de crecimiento promedio anual de $5,21 \%$ durante el periodo 2005-2014. En el año 2014 la producción total nacional registra un valor de 25.143,95 GWh (Gigavatio hora), con un aporte de Tungurahua del 1,89\% a través de EEASA.

Figura 1: Producción nacional anual de energía eléctrica Periodo 2005-2014

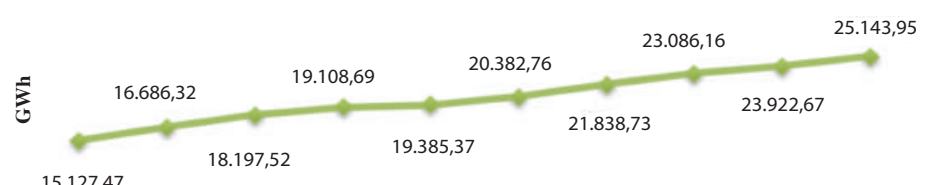

$15.127,47$

$\begin{array}{llllllllll}2005 & 2006 & 2007 & 2008 & 2009 & 2010 & 2011 & 2012 & 2013 & 2014\end{array}$

$\longrightarrow$ Producción de energía eléctrica (GWh)

Fuente: ARCONEL. Estadística multianual del sector eléctrico 2005-2014 Elaboración: Equipo OBEST

En el 2014, la producción total de energía eléctrica en Tungurahua, por parte de EEASA, se ubica en 476,27 GWh; produciéndose un incremento del $6 \%$ entre enero y diciembre; reflejando así una tasa de crecimiento mensual de 0,47\%.

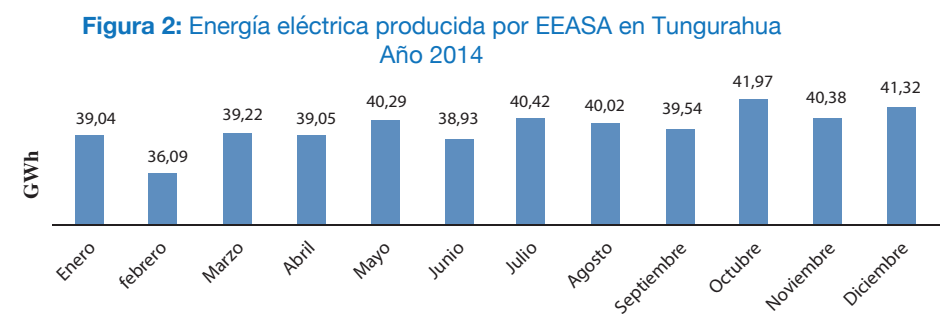

- Energía eléctrica producida por EEASA en Tungurahua (GWh)

\section{Demanda de energía eléctrica}

A nivel nacional, el grupo de mayor consumo de energía eléctrica en el 2014 es el residencial (6.364 GWh); mientras que el alumbrado público es el grupo con menor consumo (1.023,34 GWh). En el periodo 2005-2014 el grupo de consumo industrial presenta la mayor tasa de crecimiento anual $(11,34 \%)$.

Figura 3: Demanda nacional anual de energía eléctrica por grupo de consumo

Periodo 2005-2014

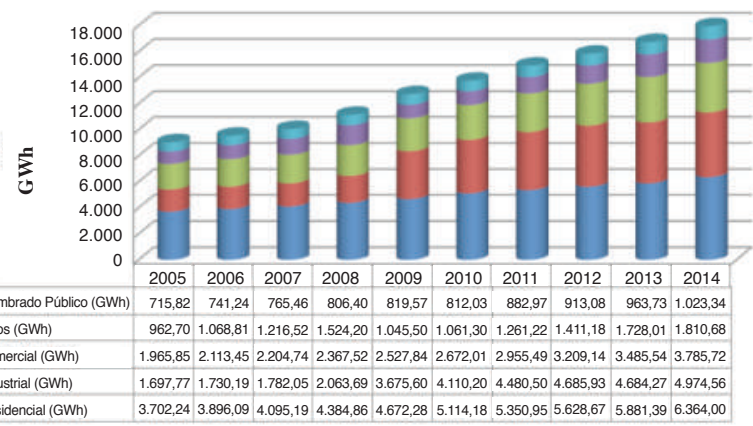

Fuente: ARCONEL. Estadística multianual del sector eléctrico 2005-2014 Elaboración: Equipo OBEST

La demanda de energía eléctrica en Tungurahua, experimenta un crecimiento a través de los años; es así que del año 2011 al 2014 pasa de 382,77 GWh a 458,15 GWh respectivamente; es decir, registra un crecimiento promedio anual de 4,59\%.

Figura 4: Demanda anual de energía eléctrica en Tungurahua Periodo 2011-2014

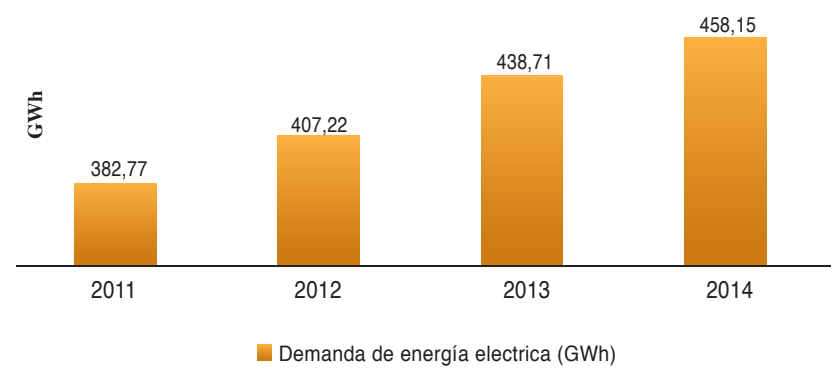

Fuente: ARCONEL. Estadística multianual del sector eléctrico 2005-2014 Elaboración: Equipo OBEST

En el 2014, Guayas registra la mayor demanda de energía eléctrica con 6.804,5 GWh, seguido por Pichincha y Manabí con 3.926,67 GWh y 1.289,6 GWh respectivamente. Por otro lado, la provincia que menos consume energía eléctrica es Galápagos con 42,09 GWh descartando las zonas no delimitadas. Tungurahua se encuentra sexta en este ranking luego de la provincia de El Oro. 
Figura 5: Demanda nacional anual de energía eléctrica por provincia Año 2014

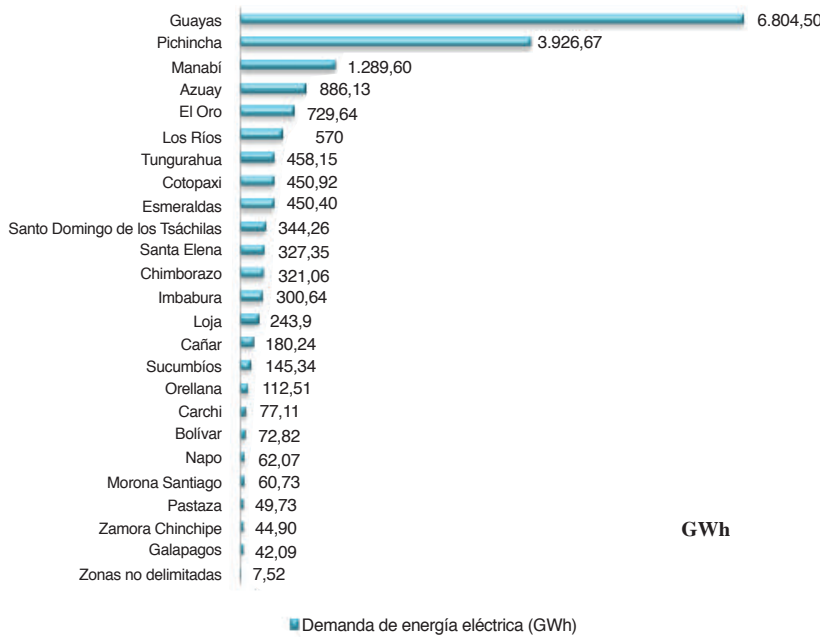

Fuente: ARCONEL. Estadística multianual del sector eléctrico 2005-2014 Elaboración: Equipo OBEST

\section{Cobertura de suministro de energía eléctrica}

La cobertura en el suministro de energía eléctrica en Ecuador, durante el periodo 2005-2014, tiene una tendencia creciente, dando a conocer que son cada vez más las personas que disponen de este servicio. En el 2005 la cobertura nacional es de 92,32\%; mientras que, para el 2014 esta aumenta a $97,04 \%$. Tungurahua por su parte, inicia el 2005 con una cobertura de 93,05\%; y para el 2014, se incrementa a 99,46\%; la variación es de 6,41 puntos, la cual supera a la obtenida a nivel nacional.

Figura 6: Cobertura de servicio eléctrico a nivel nacional y en Tungurahua Periodo 2005-2014

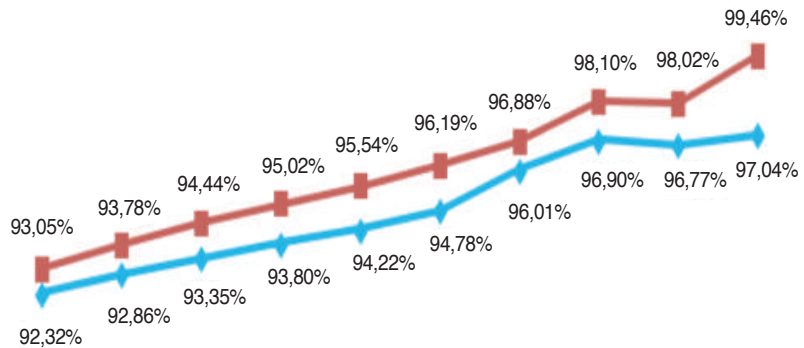

$\begin{array}{llllllllll}2005 & 2006 & 2007 & 2008 & 2009 & 2010 & 2011 & 2012 & 2013 & 2014\end{array}$

$=$ Cobertura servicio eléctrico nacional $=$ Cobertura servicio eléctrico Tungurahua

\section{Fuente: CONELEC. Indicadores de energía eléctrica anuales
Elaboración: Equipo OBEST}

Para el año 2014, Tungurahua es una de las provincias con mayor cobertura en el suministro de energía eléctrica (99,46\%), encontrándose en tercer lugar a nivel provincial, luego de Galápagos y Pichincha.
Figura 7: Cobertura de servicio eléctrico a nivel provincial Año 2014

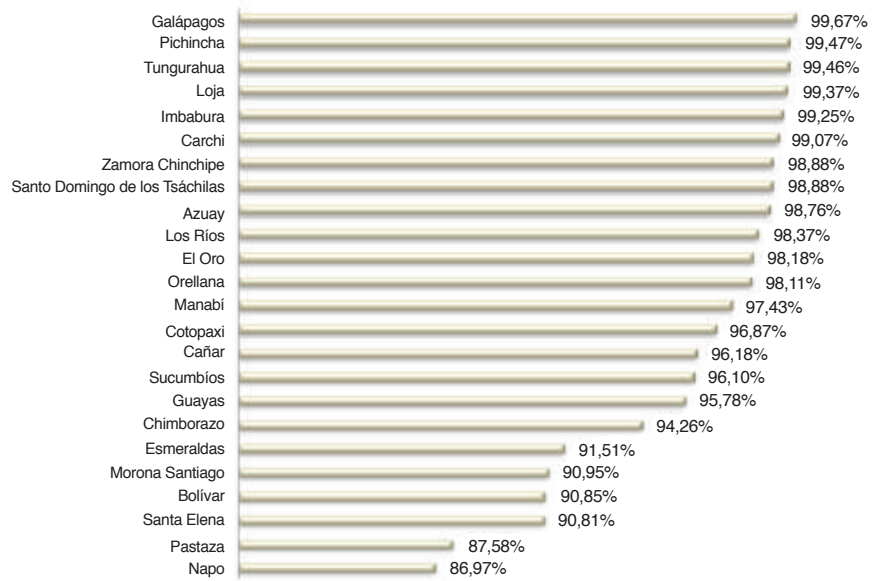

u Cobertura servicio eléctrico

Fuente: CONELEC. Indicadores de energía eléctrica anuales Elaboración: Equipo OBEST

\section{Pérdida de energía eléctrica}

Las pérdidas de energía eléctrica se constituyen en la energía que se pierde en cada una de las etapas funcionales del sistema de distribución (ARCONEL, 2015, p.59).

A nivel nacional, el porcentaje de pérdidas de energía en el sistema de distribución muestra un decrecimiento entre los años 2013-2014, pasando de $12,62 \%$ a 12,38\% respectivamente; es decir que, la disminución es de 0,24 puntos.

Figura 8: Pérdida nacional de energía eléctrica en el sistema de distribución Periodo 2013-2014

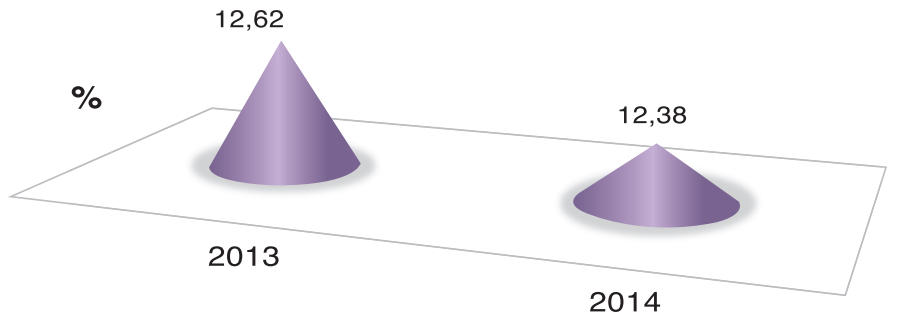

"Pérdidas Sistema (\%)

Fuente: ARCONEL. Estadistica multianual del sector eléctrico 2005-2014 Elaboración: Equipo OBEST

Del 2013 al 2014, EEASA es una de las 8 empresas a nivel nacional que registran incremento en pérdida de energía eléctrica, es así que, de $6,20 \%$ en el 2013 sube a 7,19\% en el 2014 (0,99 puntos). A pesar de esto, EEASA destaca por ser una de las empresas con menor pérdida de energía eléctrica en el Ecuador en el 2014; según datos de la misma institución, la pérdida de energía eléctrica, sólo en Tungurahua, es de $6,35 \%$ en el 2014. La organización que posee el mayor incremento de pérdida de energía, entre los años 2013 y 2014, es la Corporación Nacional de Electricidad de Esmeraldas - CNEL-Esmeraldas (1,86 puntos). 
Figura 9: Pérdida de energía eléctrica en el sistema de distribución según empresa Periodo 2013-2014

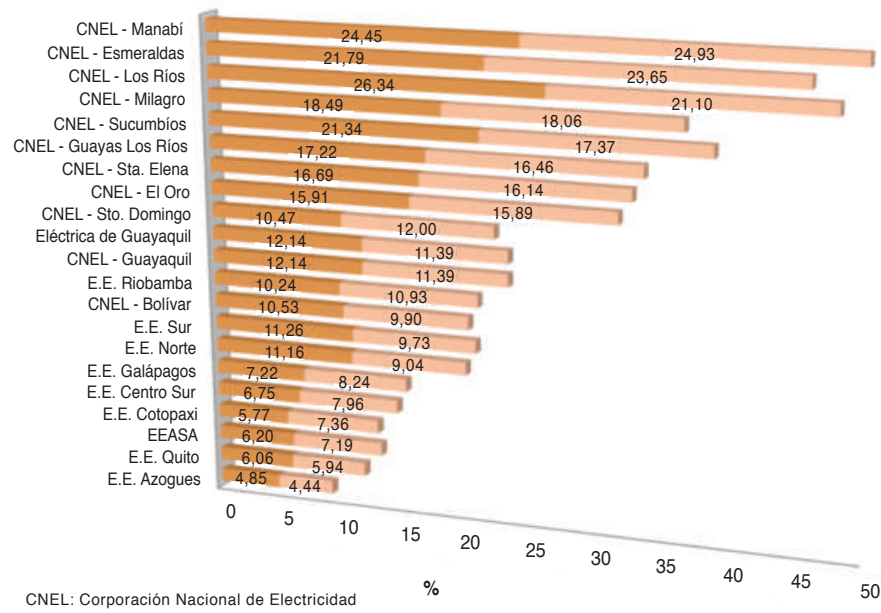

E.E.: Empresa Eléctrica

$$
\begin{array}{r}
\text { E.E.: Empresa Eléctrica } \quad \text { \#Pérdida Sistema } 2013(\%) \quad \text { " Pérdida Sistema } 2014(\%) \\
\text { Fuente: ARCONEL. Estadística multianual del sector eléctrico 2005-2014 } \\
\text { Elaboración: Equipo OBEST }
\end{array}
$$

\section{Precios medios de la energía eléctrica}

Según ARCONEL (2015), el precio medio de la energía eléctrica hace referencia a la relación promedio entre el valor de la energía en dólares (USD) y la cantidad de energía facturada en kWh (Kilovatio hora). Los precios que se analizan a continuación, son los facturados por empresas distribuidoras de electricidad a clientes regulados (aquellos cuya facturación se rige a lo dispuesto en el pliego tarifario elaborado por la ARCONEL).

A nivel nacional, los precios medios de la energía eléctrica tienen una leve tendencia a la baja en el periodo 2005-2013; es así que, de 8,82 USD \$/kWh (centavos de dólar por kilovatio hora) en el 2005, se reduce a 8,1 USD $\$ / \mathrm{kWh}$ en el 2013 , reflejando una tasa promedio de disminución anual de -0,94\%. Sin embargo, del año 2013 al 2014 , con la aplicación del nuevo pliego tarifario en mayo del 2014 , existe un incremento en los precios medios; de 8,1 USD $\$ / \mathrm{kWh}$ sube a 8,98 USD \$/kWh; la variación es de 10,86\%.

EEASA por su parte, presenta altos y bajos en sus precios medios de energía eléctrica en el periodo 2005-2014; la variación más significativa se da entre los años 2007 y 2008 ya que el precio se incrementa en $21,22 \%$. A partir del 2008 el precio medio de EEASA supera al precio medio nacional, siendo en el 2014 de 9,72 USD $\$ / \mathrm{kWh}(0,74$ USD $\$ / \mathrm{kWh}$ mayor al precio medio nacional). La tasa promedio de crecimiento anual a lo largo del periodo es de 1,70\%.

Figura 10: Precios medios de energía eléctrica facturada por empresas distribuidoras a clientes regulados, a nivel nacional y EEASA Periodo 2005-2014.

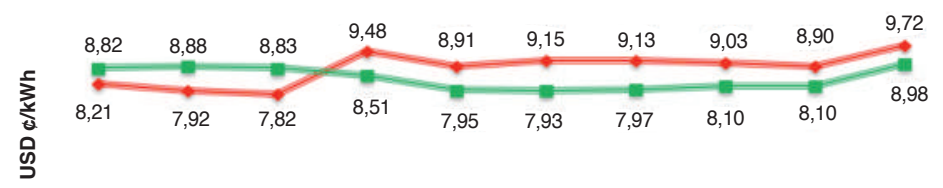

$\begin{array}{llllllllll}2005 & 2006 & 2007 & 2008 & 2009 & 2010 & 2011 & 2012 & 2013 & 2014\end{array}$

—Precio medio EEASA (USD c/kWh) = Precio medio nacional (USD c/kWh)

Fuente: ARCONEL. Estadística multianual del sector eléctrico 2005-2014 / SNI. Indicadores y datos sector electricidad
Elaboración: Equipo OBEST
Para Julio del 2015, EEASA es una de las empresas distribuidoras cuyo precio medio de energía eléctrica se encuentra en un nivel alto, el valor registrado es de 10,10 $\$ / \mathrm{kWh}$, ubicándose sexta en este ranking luego de la Empresa Eléctrica Norte - E.E. Norte. Sin embargo, el mayor precio medio lo registra la Empresa Eléctrica Sur E.E. Sur con un valor de 10,85 \$/kWh.

Figura 11: Precios medios de energía eléctrica facturada a clientes regulados según empresa distribuidora, Julio 2015.

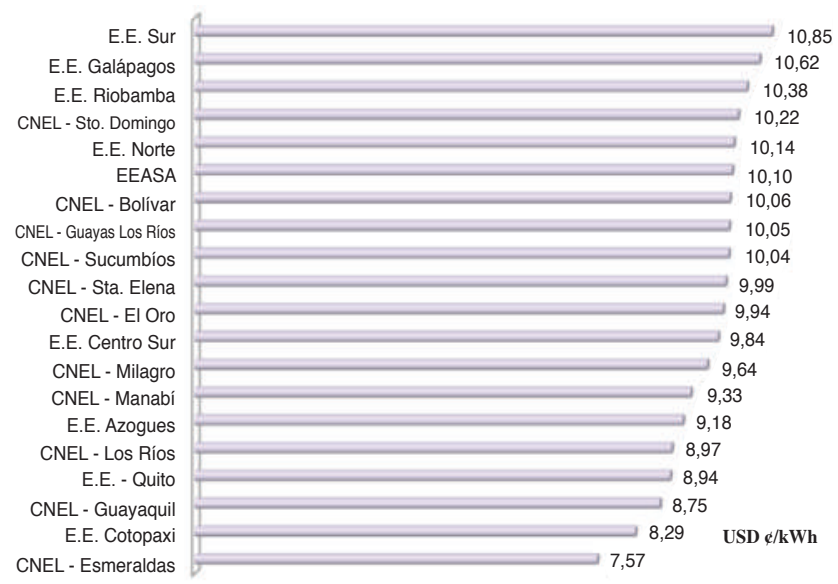

Precio medio (USD c/kWh) CNEL: Corporación Nacional de Electricidad E.E.: Empresa Eléctrica

Fuente: SNI. Indicadores y datos sector electricidad Elaboración: Equipo OBEST

Referencias:

- Agencia de Regulación y Control de Electricidad - ARCONEL. (2015). Estadística multianual del sector eléctrico 2005-2014. Recuperado de:

http://www.conelec.gob.ec/archivos_articulo/doc_10525_MultianualSectorElectricoEcuatoriano2005-2014.pdf

- Consejo Nacional de Electricidad - CONELEC. (2012). Boletín estadístico sector eléctrico ecuatoriano 2011. Recuperado de:

http://www.conelec.gob.ec/images/documentos/doc_10104_Bolet\%C3\%ADn\%20A\%C3\%B10\% 202011.pdf

- Empresa Eléctrica Ambato Regional Centro Norte S.A. - EEASA. Estadística Anual 2014. Recuperado de:

http://www.eeasa.com.ec/index.php?id =5\&id $1=46 \&$ id $2=8$

Elaboración:

Ing. Fernando Andrés Mayorga Núñez 